\title{
Unusual Case of Angle Closure Glaucoma in a Patient with Neurofibromatosis Type 1
}

\author{
Flavio Mantelli $^{a} \quad$ Solmaz Abdolrahimzadeh $^{\mathrm{b}} \quad$ Giuseppe Mannino $^{\mathrm{c}}$ \\ Alessandro Lambiase ${ }^{d}$ \\ ${ }^{a}$ IRCCS Fondazione Bietti, and ${ }^{b}$ Ophthalmology Unit, DAI Testa Collo, Azienda Policlinico \\ Umberto I, ${ }^{C}$ Ophthalmology Unit, NESMOS Department, St. Andrea Hospital, and \\ ${ }^{d}$ Ophthalmology Section, Department Organi di Senso, University of Rome Sapienza, \\ Rome, Italy
}

\section{Key Words}

Datura $\cdot$ Glaucoma $\cdot$ Neurofibromatosis

\begin{abstract}
We report the case of a 29-year-old female patient who presented with an acute onset of anisocoria, blurred vision, nausea and severe left-sided headache. There was no history of trauma, drug abuse, or instillation of topical mydriatic compounds. The ocular history was negative for similar events. On presentation, her visual acuity was 0.2 in the left and 1.0 in the right eye with $a+2.5 \mathrm{dpt}$ sph. correction. Slit-lamp examination demonstrated a shallow anterior chamber as well as the presence of iris nodules in both eyes. These nodules were identified as Lisch nodules as the patient referred to the previous diagnosis as being neurofibromatosis type 1. A third nerve palsy was considered, but a brain MRI showed normal results. Her ocular motility was normal, but the left pupil was mydriatic and poorly reacting to light, with an associated raised intraocular pressure (IOP) of $38 \mathrm{~mm} \mathrm{Hg}$. An examination of the fellow eye was normal, with the IOP measuring $18 \mathrm{~mm} \mathrm{Hg}$. Gonioscopy of the right eye showed a narrow angle. On further anamnestic investigation, the patient revealed that the pain and the blurred vision begun in the morning while she was helping her mother in the garden. Finally, after showing the patient a picture of Datura flowers, which she recognized immediately, we made the unusual diagnosis of angle closure glaucoma by Datura, a well-known toxic plant with mydriatic properties. The patient was successfully treated with systemic acetazolamide and topical pilocarpine.


Mantelli et al.: Unusual Case of Angle Closure Glaucoma in a Patient with Neurofibromatosis Type 1

\section{Introduction}

Neurofibromatosis type 1 (NF1) is an autosomal dominant disease caused by mutations in the tumor suppressor gene NF1, which results in increased cellular proliferation and leads to tumor formation [1]. There are a number of ocular manifestations associated with NF1, including iris Lisch nodules, optic nerve gliomas, neurofibromas of the eye as well as orbit and choroidal nodules [2-4].

Glaucoma onset is rarely seen in NF1, although multiple pathogenic mechanisms related to angle closure have been proposed. Among them, the most commonly reported are: (1) direct infiltration of the anterior chamber by neurofibromas that clog the angle, (2) secondary angle closure by neurofibromatous cysts or a thickening of the ciliary body and choroid, (3) neovascular glaucoma, and (4) developmental angle abnormalities [5, 6]. Nevertheless, glaucoma associated with NF remains rare, and, to our knowledge, this is the first report of acute angle closure glaucoma in NF1 with the absence of angle abnormalities.

\section{Case Report}

A 29-year-old female presented to the emergency department because of a sudden unilateral mydriasis observed by her mother, accompanied by increasing left eye discomfort in the previous hours, difficulty in near and distance vision and severe left-sided headache and nausea. No accompanying diplopia or other neurological symptoms were observed, and both vital signs and mental status were normal. A third nerve palsy or a compressive pathology was considered, and a brain MRI was performed without any abnormal results.

Upon referral to the eye unit, the patient's Snellen visual acuity with a $+2.50 \mathrm{dpt}$ sph. correction was reduced to 20/100 in the left eye, while in the right eye she showed a 20/20 vision. Slit-lamp examination of the left eye demonstrated a cornea sub-oedema, a shallow anterior chamber, and a raised intraocular pressure (IOP) of $38 \mathrm{~mm} \mathrm{Hg}$ measured by ICare tonometry [7]. The left pupil was dilated and fixed to approximately $9 \mathrm{~mm}$ and unresponsive to both light and accommodation reflexes. The left pupil did not constrict completely when pilocarpine 1\% drops were instilled (fig. 1), suggesting a pharmacological origin of the condition and excluding other conditions such as Adie's pupil. Slit lamp examination of the right eye was normal, except for the presence of Lisch iris nodules and gonioscopy showing a narrow angle (fig. 2). The consensual light reflex of the right eye was present.

Ultrasound biomicroscopy was performed to evaluate anterior chamber structures and the ciliary bodies, and it confirmed the angle closure in the left eye [8]. The presence of Lisch nodules in both eyes was also well-evidenced by ultrasound biomicroscopy imaging (fig. 3).

Upon further anamnestic investigation, the patient revealed that blurred vision and pain had begun in the morning while she was helping her mother in the garden. Knowing the toxic mydriatic effects of several plants such as Datura and Brugmansia (they contain tropane alkaloids such as scopolamine, hyoscyamine and atropine), we showed the patient images of these plants using an internet search [9]. The patient immediately recognized the Datura plant. She did not have any direct eye contact with the leaves or flowers, but expressed that she had handled both leaves and flowers with her bare hands. It is very likely that an accidental hands-eye contact after touching the plant had occurred. Therefore, the diagnosis of 'gardener's eye' or anisocoria by vegetal intoxication was made [9].

The raised IOP was partly relieved following the administration of intravenous acetazolamide $250 \mathrm{mg}$, and the topical administration of pilocarpine 4\%. Afterwards, she reported 
Mantelli et al.: Unusual Case of Angle Closure Glaucoma in a Patient with Neurofibromatosis Type 1

relief of both pain and nausea. When her IOP stabilized to $19 \mathrm{~mm} \mathrm{Hg}$, the patient was dismissed from the hospital with pilocarpine $2 \%$ t.i.d. treatment.

On the next day, with a fully restored corneal transparency, a preventive YAG-laser peripheral iridotomy was performed in both eyes. At her last follow-up visit 6 months later, her best corrected visual acuity was 20/20 in both eyes, with an IOP of $16 \mathrm{~mm}$ Hg without any anti-glaucoma medication. Fundus examination of both eyes showed a healthy optic nerve head (cup: disc ratio 0.3 ).

\section{Discussion}

This case report represents an interesting addition to the literature on the differential diagnosis of acute anisocoria in patients with NF1.

A wide range of ocular and neurological disorders need to be considered when dealing with a unilateral, dilated unresponsive pupil. In fact, many different causes are possible, from blunt trauma to an inadvertent application of topical mydriatics, Adie's pupil, third nerve palsy, and onset of acute glaucoma [10].

The differential diagnosis can be more difficult in NF patients because in this disorder, isolated palsies of the third, fourth, and sixth nerves have been reported, mostly in association with nerve schwannomas [11]. Such ophthalmoplegic attacks are usually associated with focal headache and systemic symptoms. Our patient did not show any neurological symptoms, ptosis or an ocular motility deficit related to a third nerve palsy; the cerebral MRI excluded a compressive origin of the anisocoria. The IOP increase associated with the clinical findings lead to the diagnosis of an acute glaucoma attack.

The observation of glaucoma in NF1 is not new and the presence of unilateral glaucoma in NF1 patients was already reported back in 1903 [12]. However, these cases were related to ectropion uveae or corneal endothelial overgrowth and did not present as acute angle closure glaucoma. The presence of a narrow angle in the right eye associated with the hypermetropic refraction may be the significant contributing factor that triggered the acute glaucoma attack in our patient. In addition, the history of exposure to alkaloid agents clarifies the pathogenic mechanism of the clinical findings. In fact, accidental mydriasis from exposure to plant components has been previously reported in humans and animals, but to the best of our knowledge, this is the first report of an acute glaucoma attack following accidental contact with Datura in a patient with NF1 [9, 10,13].

Datura stramonium is a common ornamental plant of the family of Solanacea, containing, primarily in their seeds and flowers, tropane alkaloids such as high levels of scopolamine (up to $0.6 \%$ dry weight), hyoscyamine and atropine [14]. Known since the 16th century, Datura has been used in preparations including intoxicants or love potions, and, more recently, as a fumigant in bronchial asthma for its relaxing effect on the smooth musculature of the bronchi and bronchioles $[13,15]$.

Systemic intoxication by ingestion has been widely described. Symptoms of Datura toxicity occur typically within $60 \mathrm{~min}$ after ingestion and continue for 24-48 h. The ingestion of 10-50 mg of atropine appears to be toxic for adults, and 4-5 mg may be fatal for small children. An intoxication produces peripheral and central neurologic symptoms that are those produced by Atropa belladonna, leading to an atropinic syndrome: tachycardia, bilateral mydriasis, dryness of the mouth and delirium. Death may result from coma, cardiac or respiratory arrest [15].

Local instillation into the eye results in mydriasis and cycloplegia by acting on the sphincter pupillae and the ciliary muscle. In fact, the alkaloids contained in the plant extract 
Mantelli et al.: Unusual Case of Angle Closure Glaucoma in a Patient with Neurofibromatosis Type 1

block peripheral parasympathetic activity by preventing the entrance of acetylcholine into the effector cells, resulting in paralysis of the smooth muscles. The severity and duration of these effects (they may vary from a few hours to several days) depend on the amount and on the duration of the exposure to alkaloids $[9,10]$.

\section{Conclusions}

We presented the case of an unusual cause of angle closure glaucoma in a patient with NF1 in order to underline the importance of a deeply conducted medical history. The uniqueness of our case was that the patient had a glaucoma attack without direct contact with the Datura flower and her eye. The underlying NF and the characteristic of the iriscorneal angle were probably important contributing factors. Since acute angle closure glaucoma is associated with headache and systemic symptoms such as nausea and vomiting, a delay in the proper diagnosis is possible, especially if coupled with a history of NF.

Therefore, this report emphasizes the importance of an accurate and detailed medical history in patients with unilateral mydriasis, regardless of known associated systemic diseases. This will avoid unnecessary and expensive diagnostic tests, as in the case of our patient, who was diagnosed with a glaucoma attack due to pharmacologic mydriasis secondary to unintentional contact with Datura.

To conclude, we want to emphasize the importance of information directed to gardening enthusiasts and garden suppliers about the potential adverse effects after handling some ornamental plants.

\section{Disclosure Statement}

The authors declare that they have no conflicts of interest.

\section{References}

1 Ferner RE, Gutmann DH: Neurofibromatosis type 1 (NF1): diagnosis and management. Handb Clin Neurol 2013;115:939-955.

-2 Richetta A, Giustini S, Recupero SM, Pezza M, Carlomagno V, Amoruso G, Calvieri S: Lisch nodules of the iris in neurofibromatosis type 1. J Eur Acad Dermatol Venereol 2004;18:342-344.

3 Recupero SM, Plateroti R, Abdolrahimzadeh S, De Dominicis M, Giustini S, Noviello MR, Calvieri S: Lisch nodules in neurofibromatosis type 1: relationship to age and cutaneous neurofibromas. Ann Ophthalmol 1996;28:178-183.

4 Jett K, Friedman JM: Clinical and genetic aspects of neurofibromatosis 1. Genet Med 2010;12:1-11.

5 Emre S, Palamar M, Ulusoy MO, Gençoğlan G: Ciliary body cysts in neurofibromatosis: a new coexistence? Graefes Arch Clin Exp Ophthalmol 2012;250:857-861.

-6 Al Freihi SH, Edward DP, Nowilaty SR, Abouammoh MA, Morales J: Iris neovascularization and neovascular glaucoma in neurofibromatosis type 1: report of 3 cases in children. J Glaucoma 2013;22:336-341.

-7 Scuderi GL, Cascone NC, Regine F, Perdicchi A, Cerulli A, Recupero SM: Validity and limits of the rebound tonometer (ICare ${ }^{\circledR}$ ): clinical study. Eur J Ophthalmol 2011;21:251-257.

-8 Mannino G, Malagola R, Abdolrahimzadeh S, Villani GM, Recupero SM: Ultrasound biomicroscopy of the peripheral retina and the ciliary body in degenerative retinoschisis associated with pars plana cysts. Br J Ophthalmol 2001;85:976-982.

-9 Raman SV, Jacob J: Mydriasis due to Datura inoxia. Emerg Med J 2005;22:310-311.

10 Macchiaiolo M, Vignati E, Gonfiantini MV, Grandin A, Romano MT, Salata M, Valentini D, Villani A: An unusual case of anisocoria by vegetal intoxication: a case report. Ital J Pediatr 2010;36:50.

11 Barrett VJ, Tan MH, Elston JS: Recurrent third nerve palsy as the presenting feature of neurofibromatosis 2. J Neuroophthalmol 2012;32:329-331. 


\begin{tabular}{l|l}
\hline Case Rep Ophthalmol 2014;5:386-391 \\
\hline DOI: 10.1159/000369334 & $\begin{array}{l}\text { @ 2014 S. Karger AG, Basel } \\
\text { www.karger.com/cop }\end{array}$ \\
\hline
\end{tabular}

Mantelli et al.: Unusual Case of Angle Closure Glaucoma in a Patient with Neurofibromatosis Type 1

12 Edward DP, Morales J, Bouhenni RA, Patil J, Edward PR, Cummings TJ, Chaudhry IA, Alkatan H: Congenital ectropion uvea and mechanisms of glaucoma in neurofibromatosis type 1: new insights. Ophthalmology 2012;119:1485-1494.

13 Hansen P, Clerc B: Anisocoria in the dog provoked by a toxic contact with an ornamental plant: Datura stramonium. Vet Ophthalmol 2002;5:277-279.

$>14$ Wilhelm H, Wilhelm B, Schiefer U: Mydriasis caused by plant contact (in German). Fortschr Ophthalmol 1991;88:588-591.

15 Gowdy J: Datura stramonium intoxication review of symptomatology in 212 cases. JAMA 1972;221:585587.

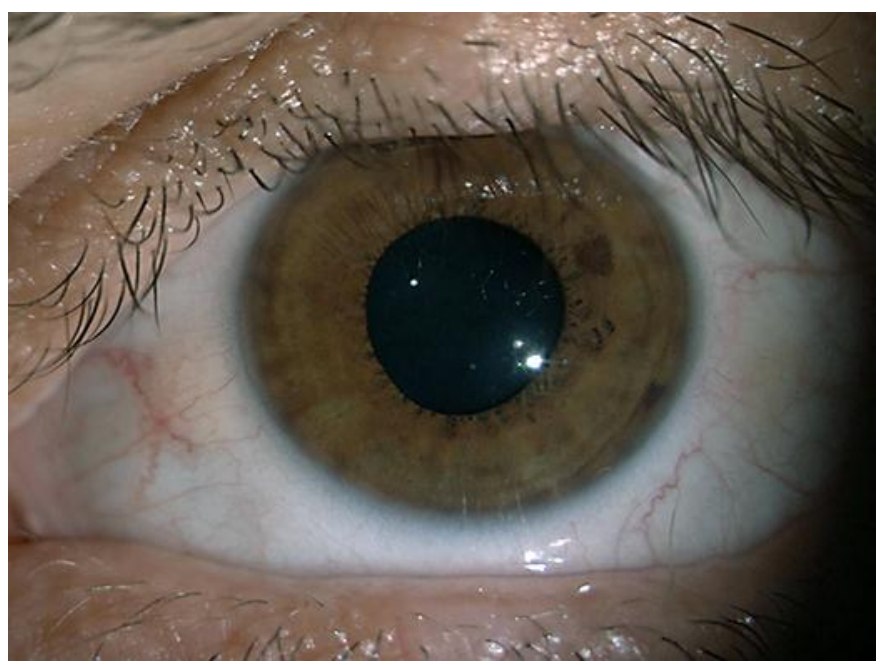

Fig. 1. Unilateral mydriasis that did not resolve completely following pilocarpine $1 \%$ eye drop administration.
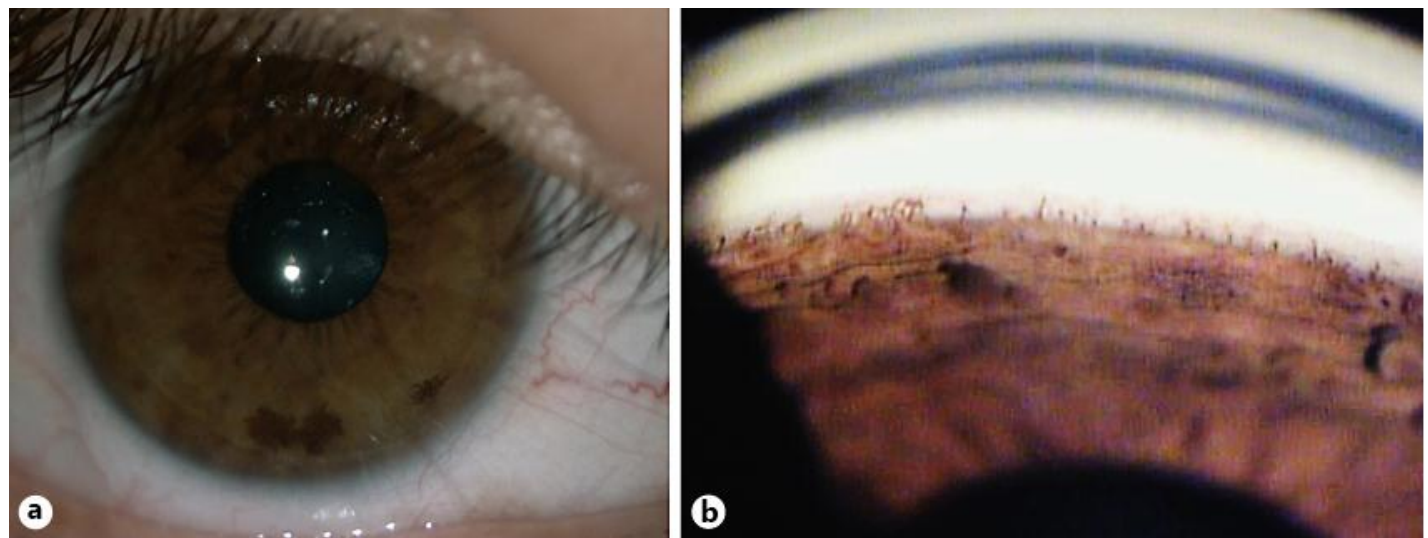

Fig. 2. Lisch iris nodules were present in both eyes of our patient (a) and gonioscopy revealed the presence of an abnormally narrow angle (b). 
Case Reports in

Ophthalmology
Case Rep Ophthalmol 2014;5:386-391

DOI: $10.1159 / 000369334$

Mantelli et al.: Unusual Case of Angle Closure Glaucoma in a Patient with Neurofibromatosis Type 1

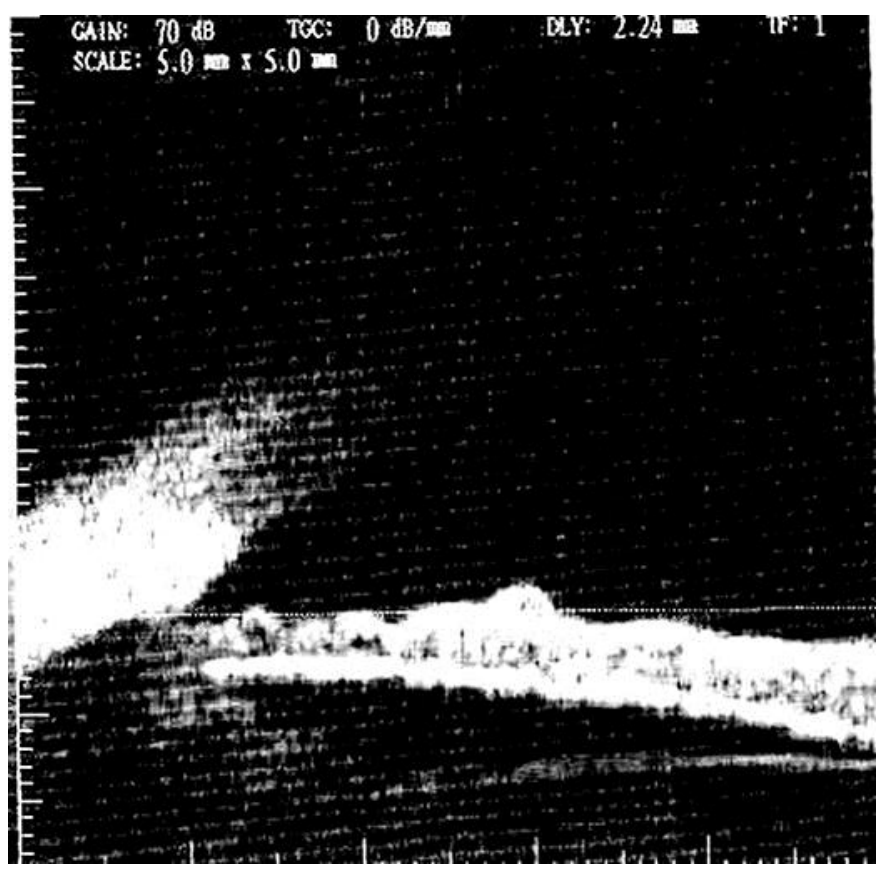

Fig. 3. Lisch iris nodules and narrow angle in our patient as evidenced by ultrasound biomicroscopy. 